\title{
Introductions and early spread of SARS-CoV-2 in the New York City area
}

\begin{abstract}
Ana S. Gonzalez-Reiche ${ }^{*}$, Matthew M. Hernandez ${ }^{2,3 *}$, Mitchell J. Sullivan', Brianne Ciferri', Hala Alshammary ${ }^{2}$, Ajay Obla ${ }^{1}$, Shelcie Fabre ${ }^{4}$, Giulio Kleiner ${ }^{2}$, Jose Polanco ${ }^{1,2}$, Zenab Khan'1, Bremy Alburquerque ${ }^{1,3}$, Adriana van de Guchte', Jayeeta Dutta', Nancy Francoeur ${ }^{1}$, Betsaida Salom Melo ${ }^{1,5}$, Irina Oussenko ${ }^{1,5}$, Gintaras Deikus ${ }^{1,5}$, Juan Soto ${ }^{1,5}$, Shwetha Hara Sridhar ${ }^{1,5}$, Ying-Chih Wang ${ }^{1,5}$, Kathryn Twyman ${ }^{6}$, Andrew Kasarskis, 1,5,6, Deena R. Altman',7, Melissa Smith ${ }^{1}$, Robert Sebra',5,8,9, Judith Aberg 7 , Florian Krammer ${ }^{2,10}$, Adolfo García-Sastre ${ }^{2,7,10,11, ~ M a r t a ~}$

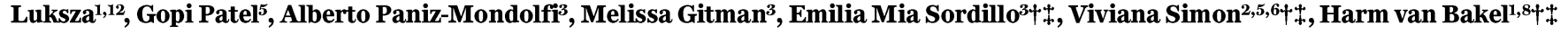
'Department of Genetics and Genomic Sciences, Icahn School of Medicine at Mount Sinai, New York, NY 10029, USA. 2Department of Microbiology, Icahn School of Medicine at Mount Sinai, New York, NY 10029, USA. ${ }^{3}$ The Graduate School of Biomedical Sciences, Icahn School of Medicine at Mount Sinai, New York, NY 10029, USA. ${ }^{4} \mathrm{Clinical}$ Microbiology Laboratory, Department of Pathology, Molecular, and Cell-Based Medicine, Icahn School of Medicine at Mount Sinai, New York, NY 10029, USA. ${ }^{2}$ cahn Institute for Data Science and Genomic Technology, Icahn School of Medicine at Mount Sinai, New York, NY 10029, USA. ${ }^{6}$ The Mount Sinai Data Office, Icahn School of Medicine at Mount Sinai, New York, NY 10029, USA. Division of Infectious Diseases, Department of Medicine, Icahn School of Medicine at Mount Sinai, New York, NY 10029, USA. ${ }^{8}$ Black

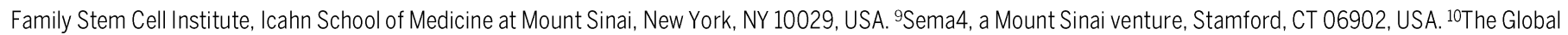
Health and Emerging Pathogens Institute, Icahn School of Medicine at Mount Sinai, New York, NY 10029, USA. 11The Tisch Cancer Institute, Icahn School of Medicine at Mount Sinai, New York, NY 10029, USA. ${ }^{12}$ Department of Oncological Sciences, Icahn School of Medicine at Mount Sinai, New York, NY 10029, USA.
\end{abstract}

*These authors contributed equally to this work.

†Corresponding author. Email: harm.vanbakel@mssm.edu (H.v.B.); viviana.simon@mssm.edu (V.S.); emilia.sordillo@mssm.edu (E.M.S.)

$\ddagger$ These authors contributed equally to this work.

New York City (NYC) has emerged as one of the epicenters of the current SARS-CoV-2 pandemic. To identify the early transmission events underlying the rapid spread of the virus in the NYC metropolitan area, we sequenced the virus causing COVID-19 in patients seeking care at the Mount Sinai Health System. Phylogenetic analysis of 84 distinct SARS-CoV2 genomes indicates multiple, independent but isolated introductions mainly from Europe and other parts of the United States. Moreover, we find evidence for community transmission of SARS-CoV-2 as suggested by clusters of related viruses found in patients living in different neighborhoods of the city.

Severe acute respiratory syndrome coronavirus 2 (SARSCoV-2; previously known as $2019-\mathrm{nCoV}$ ) is an emerging viral pathogen that was first reported to cause severe respiratory infections in Wuhan, China in late December 2019. Over the past months, it rapidly spread across the globe and the World Health Organization (WHO) declared a pandemic on March 11, 2020. Targeted screening of suspected coronavirus disease 2019 (COVID-19) cases, as well as a series of successive nation-wide travel restrictions were put in place to curtail SARS-CoV-2 introductions into the USA from outbreak hotspots in China (February 2, 2020), Iran (March 2, 2020); mainland European countries (March 13, 2020), and the UK and Ireland (March 16, 2020) to the continental United States (1-4). Despite these measures, the first COVID-19 case in New York State was identified in New York City (NYC) on February 29, 2020. During the first weeks of March, the number of cases rapidly increased, due to expansion of screening capacity following implementation of automated platforms for detection of SARS-CoV-2 infections by local health system diagnostic laboratories and reference laboratories. As of May 2, 2020, there were 312,977 confirmed COVID-19 cases in NY state, including 172,354 (55\%) in NYC (NY State Department of Health (NYS DOH); https://coronavirus.health.ny.gov/). With more than 13,300 fatalities in the metropolitan area, NYC has been one of the major epicenters of SARS-CoV-2 infections in the USA.

The Pathogen Surveillance Program (PSP) at the Icahn School of Medicine at Mount Sinai is a multidisciplinary, institutional infrastructure which seeks to generate high resolution, near real-time genetic information on pathogens found to cause disease in the large and diverse patient population seeking care at the Mount Sinai Health System in NYC. Following biospecimen coding, nucleic acid extraction and qPCR quantification, next-generation sequencing approaches based on Illumina and Pacific Biosciences technology provide information on the pathogen's genome. The process has been optimized for quick turnaround, optimized data assembly and, last but not least, integration with de-identified demographic information.

We took advantage of the existing PSP infrastructure to investigate the origins of SARS-CoV-2 strains circulating in NYC and to dissect the spread of the virus in this metropolitan area with a high-density population. Here, we present the genomic diversity of 90 SARS-CoV-2 isolates obtained from 
84 patients seeking care at the Mount Sinai Health System between February 29, 2020 and March 18, 2020. These genomes provide clear evidence for multiple, independent SARS-CoV-2 introductions into NYC during the first weeks of March 2020. Based on genetic similarity and phylogenetic analysis of full-length viral genome sequences, most cases diagnosed during the 18 days after the first-reported COVID-19 case in NY State appear to be associated with untracked transmission and potential travel-related exposures. Notably, the majority of introductions appear to have been sourced from Europe and the USA. We also identified two clusters totaling 21 closely related cases suggesting community spread. These observations are also supported by the city-wide distribution of these cases, which mapped to three of four represented NYC boroughs and five NY State Counties. Our data point to the limited efficacy of travel restrictions into NYC for preventing spread in the metropolitan area once multiple introductions of SARS-CoV-2 and community-driven transmission had already occurred.

Initial diagnostic testing for SARS-CoV-2 in NYC was targeted and limited to individuals fitting a set of criteria outlined by the Centers for Disease Control and Prevention (CDC), and requiring pre-approval by the local Department of Health. Between March 9-14, 2020 screening capacity at the Mount Sinai Health System (MSHS) was greatly expanded, leading to a surge of newly diagnosed cases (Fig. 1). Within one week, the number of daily positive SARS-CoV-2 tests exceeded the normal volume of positive tests for influenza virus by a factor of 5 . We sequenced 90 SARS-CoV-2 genomes from clinical isolates obtained from 84 of the $>800$ cases identified up to March 18, yielding 72 complete and 18 partial ( $>95 \%$ coverage) genomes. These cases were drawn from 21 NYC neighborhoods across four boroughs (Manhattan, Bronx, Queens and Brooklyn), as well as two towns in Westchester County. Sequenced isolates were obtained directly from nasopharyngeal swab samples collected from 44 females $(52.4 \%)$ and $40(47.7 \%)$ males ranging in age between $20-44$ years (16\%), $45-64$ years (33\%), 65-79 years $(27.4 \%)$, and $>80(16.7 \%)$ years. Of the 68 cases with available hospital visit information, 12 were discharged (17.6\%), 53 were admitted (80\%), and three were initially discharged and later admitted on a subsequent visit (2.4\%).

We performed phylogenetic analysis of the 84 unique patient isolates, together with 2,363 sequences deposited in GISAID up to April 1, 2020 (Fig. 2A). NYC isolates were distributed throughout the phylogenetic tree; consistent with multiple independent introductions. We assigned each SARS$\mathrm{CoV}-2$ isolate derived from a patient seeking care at the Mount Sinai Health System to one of the main monophyletic clades based on amino acid and nucleotide substitutions and statistical support from both maximum-likelihood (ML) and Bayesian methods. These mutations were used only for the purpose of classification, as the functional impact of many of these substitutions remains unknown. As a reference, we adopted the clade nomenclature from the NextStrain tool (5, 6 ). For each assigned clade, we identified different types of events according to the position of the NYC sequences and available epidemiological information (Table 1). The first isolate was obtained from a patient with documented exposure through travel to the Middle East (clade A3, node 5), and the second from a patient with documented travel to Europe (clade B, node 6). We therefore excluded these two cases from any inference made from the phylogenetic analyses. For the remaining isolates, the great majority (87\%) clustered with clade A2a. This clade is largely composed of isolates obtained from patients with COVID-19 in Europe (72\%) (Fig. 2B), suggesting that introductions from Europe account for the majority of cases found in NYC in the first weeks of March 2020.

Most of the NYC isolates within clade A2a were interspersed and we did not observe grouping by country or geographical region (fig. S1). Despite their diverse origins, many sequences in this clade are highly similar or identical, which makes it impossible to resolve direct relationships or directionality between cases without additional epidemiological data. We next used ML and Bayesian phylodynamic analysis to infer the time of virus transmission events related to NYC (fig. S2A). For clade A2a, we estimated a period of untracked global transmission from late January to mid-February, consistent with epidemiological observations of the developing pandemic (Table 1, node 2, and fig. S2A). The earliest sequences at the base of clade A2a include isolates from Italy, Finland, Spain, France, the UK, and other European countries from late February, in addition to a few North American isolates (Canada and US) from the first week of March 2020. Within this clade, we identified two mutations that distinguish clusters of sequences from NYC and elsewhere, suggestive of at least two independent introductions that likely occurred in early to mid-February (Table 1, nodes 3 to 4 , and fig. S2A). Notably, one of these two clusters contained 64 of the 72 NYC isolates positioned in clade A2a, suggestive of local spread (see below).

Similar to isolates in clade A2a, SARS-CoV-2 isolates in our study positioned in clade Ala (6\%) were interspersed among isolates from multiple regions with unknown directionality (Fig. 2A, node 1). This clade is also largely composed of European-origin isolates (82\%) (Fig. 2B and fig. S1).

For the rest of the clades (B, B1, and B4), we identified four putative SARS-CoV-2 virus introductions to NYC sometime between February and early March (Table 1, nodes 7 to 10, and fig. S2A). Notably, two of these introductions were inferred to be of domestic origin based on their close relationship with US isolates, including those from the main community transmission in Washington state (Clade B1, node 9) (7). The introduction of this clade to the East Coast was 
recently reported (8). Although more than half of the sequences in clade B were of Asian origin (Fig. 2B) the closest relatives to the $\mathrm{NY}$ isolates were of European and North American origin. The isolate that belongs to clade $\mathrm{B} 4$ is within a node with an inferred date of early March and a prior period of untracked transmission in unknown location(s) during February (Table 1, node 10, and fig. S2A). Prior to this period, the closest viral isolates basal to this cluster are from Australia and China (fig. S1).

The sequenced isolates and assigned clades were spatially distributed throughout all NYC boroughs and 21 neighborhoods (Fig. 3). Isolates sequenced at other NYC hospitals collected within a comparable time window had a similar clade and spatial distribution (9).

Taken together, our results show that the NYC SARS-CoV2 epidemic has been mainly sourced from untracked transmission between the US and Europe, with limited evidence of direct introductions from China where the virus originated.

Despite the relatively small number of SARS-CoV-2 sequences available in early April 2020, we identified two monophyletic clusters positioned within clade A2a that almost exclusively contained isolates from NY (Fig. 2B). One cluster included $23 \%$ (17) of the isolates contained in clade A2a and $20 \%$ of the total isolates sequenced. Based on zip code information, the cases from this cluster were distributed across five counties, including one sample from New Rochelle, Westchester County, which is part of the metropolitan area directly north of NYC and reported the first documented cluster of community-acquired infections in NY State on March 3,2020 . This cluster was characterized by the amino acid substitution A1844V in the ORF1b gene. Basal to these clusters are isolates from the states of $\mathrm{MN}, \mathrm{WA}$, and $\mathrm{CA}$. The relatedness of other US and NY isolates suggests that viruses spreading locally could have been introduced to NY through a domestic route (fig. S1).

The second cluster was a smaller group that contained four isolates from Manhattan/NYC and one isolate from Queens/NYC. Zip code information was available for three of the Manhattan cases, which were mapped to three different neighborhoods further supporting community spread. Although most NYC cases are intermixed within this largely European clade, these results suggest that domestic introductions may have also been a source of early community spread within NYC.

SARS-CoV-2 is the cause of one of the largest non-influenza pandemics of this century. Sequencing of SARS-CoV-2 isolates from 84 patients diagnosed with COVID19 at one of the largest healthcare systems in NYC during the first weeks of March 2020 provides insights into the origin and diversity of this new viral pathogen in the region. We find clear evidence for multiple independent introductions into the NYC metropolitan area from different regions globally, as well as from other parts of the United States. Our data indicates that early introductions by cases that were identified based on their known travel histories did not seed the larger community clusters, suggesting that their early quarantine and hospitalization were effective in curtailing further spread. With increased testing, we observed the emergence of communityacquired infections. Most of these were caused by viral isolates derived from clades that were circulating in Europe, likely reflecting local transmissions from undetected introductions.

A limitation of our analysis is the relatively small number of global isolates from cases identified in the first weeks of March 2020, which means that our model relies on inferences based on available background sequences at that time. Thus, some of our inferences may change as more complete and representative SARS-CoV-2 sequences become available. Moreover, since global sequencing efforts are disparate, the fraction of sequences available by region/country is not necessarily representative of the number of cases reported for each of these regions. As shown by our estimates from different methods and molecular clock models, tMRCA estimates and precision can vary even with the same data. Finally, in the absence of detailed epidemiological data on travel history and contacts, we were not able to associate periods of untracked transmissions with any specific regions/countries.

Taken together, we provide a first analysis of the SARSCoV-2 viral genotypes collected from patients seeking medical care in the NYC metropolitan area. We find that New York City, as an international hub, provides not only a snapshot of the diversity of disease-causing SARS-CoV-2 at the global level but also informs on the dynamics of the pandemic at the local level. Future studies as well as additional sequencing are needed to define viral phenotypes and further explore the evolution of the SARS-CoV-2 epidemic in NYC, as well as assess the impact of the public health measures on community transmission.

\section{REFERENCES AND NOTES}

1. Executive Office of the President, Proclamation No. 9984, 85 F.R. 6709 (2020).

2. Executive Office of the President, Proclamation No. 9992, 85 F.R. 12855 (2020).

3. Executive Office of the President, Proclamation No. 9993, 85 F.R. 15045 (2020).

4. Executive Office of the President, Proclamation No. 9996, 85 F.R. 15341 (2020).

5. J. Hadfield, C. Megill, S. M. Bell, J. Huddleston, B. Potter, C. Callender, P. Sagulenko, T. Bedford, R. A. Neher, Nextstrain: Real-time tracking of pathogen evolution. Bioinformatics 34, 4121-4123 (2018). doi:10.1093/bioinformatics/bty407 Medline

6. T. Bedford, R. Neher, J. Hadfield, E. Hodcroft, T. Sibley, J. Huddleston, J. Lee, K. Fay, S. Bell, C. Megill, B. Potter, P. Sagulenko, C. Callender, M. Ilcisin, L. Moncla, A. Black, A. Brito, N. Grubaugh, Nextstrain build for novel coronavirus SARS-CoV-2 (Github, 2020; https://github.com/nextstrain/ncov).

7. T. Bedford, A. L. Greninger, P. Roychoudhury, L. M. Starita, M. Famulare, M.-L. Huang, A. Nalla, G. Pepper, A. Reinhardt, H. Xie, L. Shrestha, T. N. Nguyen, A. Adler, E. Brandstetter, S. Cho, D. Giroux, P. D. Han, K. Fay, C. D. Frazar, M. Ilcisin, K. Lacombe, J. Lee, A. Kiavand, M. Richardson, T. R. Sibley, M. Truong, C. R. Wolf, D. A. Nickerson, M. J. Rieder, J. A. Englund, the Seattle Flu Study Investigators, J. 
Hadfield, E. B. Hodcroft, J. Huddleston, L. H. Moncla, N. F. Müller, R. A. Neher, X. Deng, W. Gu, S. Federman, C. Chiu, J. Duchin, R. Gautom, G. Melly, B. Hiatt, P. Dykema, S. Lindquist, K. Queen, Y. Tao, A. Uehara, S. Tong, D. MacCannell, G. L. Armstrong, G. S. Baird, H. Y. Chu, K. R. Jerome, Cryptic transmission of SARSCoV-2 in Washington State. medRxiv 2020.04.02.20051417 [Preprint] 16 April 2020). https://doi.org/10.1101/2020.04.02.20051417.

8. J. R. Fauver, M. E. Petrone, E. B. Hodcroft, K. Shioda, H. Y. Ehrlich, A. G. Watts, C. B. F. Vogels, A. F. Brito, T. Alpert, A. Muyombwe, J. Razeq, R. Downing, N. R. Cheemarla, A. L. Wyllie, C. C. Kalinich, I. M. Ott, J. Quick, N. J. Loman, K. M. Neugebauer, A. L. Greninger, K. R. Jerome, P. Roychoudhury, H. Xie, L. Shrestha, M.-L. Huang, V. E. Pitzer, A. Iwasaki, S. B. Omer, K. Khan, I. I. Bogoch, R. A. Martinello, E. F. Foxman, M. L. Landry, R. A. Neher, A. I. Ko, N. D. Grubaugh, Coastto-Coast Spread of SARS-CoV-2 during the Early Epidemic in the United States. Cell S0092-8674(20)30484-0 (2020). doi:10.1016/i.cell.2020.04.021 Medline

9. M. T. Maurano, S. Ramaswami, G. Westby, P. Zappile, D. Dimartino, G. Shen, X. Feng, A. M. Ribeiro-dos-Santos, N. A. Vulpescu, M. Black, M. Hogan, C. Marier, P. Meyn, Y. Zhang, J. Cadley, R. Ordonez, R. Luther, E. Huang, E. Guzman, A. Serrano, B. Belovarac, T. Gindin, A. Lytle, J. Pinnell, T. Vougiouklakis, L. Boytard, J. Chen, L. H. Lin, A. Rapkiewicz, V. Raabe, M. I. Samanovic-Golden, G. Jour, I. Osman, M. Aguero-Rosenfeld, M. J. Mulligan, P. Cotzia, M. Snuderl, A. Heguy, Sequencing identifies multiple, early introductions of SARS-CoV2 to New York City Region. medRxiv 2020.04.15.20064931 [Preprint] 23 April 2020). https://doi.org/10.1101/2020.04.15.20064931.

10. mjsull, mjsull/COVID_pipe: initial release. Zenodo (2020); doi:10.5281/zenodo.3775031.

11. J. Quick, N. D. Grubaugh, S. T. Pullan, I. M. Claro, A. D. Smith, K. Gangavarapu, G. Oliveira, R. Robles-Sikisaka, T. F. Rogers, N. A. Beutler, D. R. Burton, L. L. LewisXimenez, J. G. de Jesus, M. Giovanetti, S. C. Hill, A. Black, T. Bedford, M. W. Carroll, M. Nunes, L. C. Alcantara Jr., E. C. Sabino, S. A. Baylis, N. R. Faria, M. Loose, J. T. Simpson, 0. G. Pybus, K. G. Andersen, N. J. Loman, Multiplex PCR method for MinION and Illumina sequencing of Zika and other virus genomes directly from clinical samples. Nat. Protoc. 12, 1261-1276 (2017). doi:10.1038/nprot.2017.066 Medline

12. M. Martin, Cutadapt removes adapter sequences from high-throughput sequencing reads. EMBnet J.17, 10-12 (2011). doi:10.14806/ej.17.1.200

13. H. Li, Minimap2: Pairwise alignment for nucleotide sequences. Bioinformatics 34 3094-3100 (2018). doi:10.1093/bioinformatics/bty191 Medline

14. B. J. Walker, T. Abeel, T. Shea, M. Priest, A. Abouelliel, S. Sakthikumar, C. A. Cuomo, Q. Zeng, J. Wortman, S. K. Young, A. M. Earl, Pilon: An integrated tool for comprehensive microbial variant detection and genome assembly improvement. PLOS ONE 9, e112963 (2014). doi:10.1371/journal.pone.0112963 Medline

15. T. Seemann, Prokka: Rapid prokaryotic genome annotation. Bioinformatics 30 , 2068-2069 (2014). doi:10.1093/bioinformatics/btu153 Medline

16. K. Katoh, D. M. Standley, MAFFT multiple sequence alignment software version 7: Improvements in performance and usability. Mol. Biol. Evol. 30, 772-780 (2013). doi:10.1093/molbev/mst010 Medline

17. L.-T. Nguyen, H. A. Schmidt, A. von Haeseler, B. Q. Minh, IQ-TREE: A fast and effective stochastic algorithm for estimating maximum-likelihood phylogenies. Mol. Biol. Evol. 32, 268-274 (2015). doi:10.1093/molbev/msu300 Medline

18. D. T. Hoang, O. Chernomor, A. von Haeseler, B. Q. Minh, L. S. Vinh, UFBoot2: Improving the Ultrafast Bootstrap Approximation. Mol. Biol. Evol. 35, 518-522 (2018). doi:10.1093/molbev/msx281 Medline

19. A. Rambaut, T. T. Lam, L. Max Carvalho, O. G. Pybus, Exploring the temporal structure of heterochronous sequences using TempEst (formerly Path-O-Gen). Virus Evol. 2, vew007 (2016). doi:10.1093/ve/vew007Medline

20. P. Sagulenko, V. Puller, R. A. Neher, TreeTime: Maximum-likelihood phylodynamic analysis. Virus Evol. 4, vex042 (2018). doi:10.1093/ve/vex042 Medline

21. J. F. C. Kingman, The coalescent. Stochastic Process. Appl. 13, 235-248 (1982). doi:10.1016/0304-4149(82)90011-4

22. M. A. Suchard, P. Lemey, G. Baele, D. L. Ayres, A. J. Drummond, A. Rambaut, Bayesian phylogenetic and phylodynamic data integration using BEAST 1.10. Virus Evol. 4, vey016 (2018). doi:10.1093/ve/vey016 Medline

23. A. J. Drummond, S. Y. W. Ho, M. J. Phillips, A. Rambaut, Relaxed phylogenetics and dating with confidence. PLOS Biol. 4, e88 (2006). doi:10.1371/journal.pbio.0040088 Medline
24. A. Rambaut, A. J. Drummond, D. Xie, G. Baele, M. A. Suchard, Posterior Summarization in Bayesian Phylogenetics Using Tracer 1.7. Syst. Biol. 67, 901904 (2018). doi:10.1093/sysbio/syy032 Medline

25. A. Rambaut, FigTree 1.4. 2 software. Institute of Evolutionary Biology, Univ. Edinburgh (2014).

\section{ACKNOWLEDGMENTS}

We are greatly indebted to our clinical colleagues for their dedication and courage in providing continued high-quality medical care under very difficult conditions. We also gratefully acknowledge the authors, originating and submitting laboratories of sequences from GISAID's EpiFlu and EpiCoV (www.gisaid.org) that were used as background for our phylogenetic inferences. We thank Denis Ruchnewitz, Michael Lässig, Martha Nelson and Nidia Trovao for their input on the phylogenetic analyses. Funding: The Research reported in this paper was supported by the Office of Research Infrastructure of the National Institutes of Health (NIH) under award numbers S100D018522 and S100D026880 as well as institutional and philanthropic funds. Protocols established for this study were in part based on influenza virus sequencing protocols established with the support of CRIP (Center for Research on Influenza Pathogenesis), an NIH funded Center of Excellence for Influenza Research and Surveillance (CEIRS, contract number HHSN272201400008C). The content is solely the responsibility of the authors and does not necessarily represent the official views of the $\mathrm{NIH}$. Author contributions: J.A., G.P., A.PM., M.G., and E.M.S. provided clinical samples for the study. D.R.A., and J.A. performed clinical evaluations. M.M.H., S.F., J.P., D.R.A., G.P., A.PM., M.G., E.M.S., V.S., and H.B. were involved in clinical sample accessioning. M.M.H., H.A., and G.K. performed RNA extraction and measured viral titers. A.S.GR., Z.K., B.A., A.G., J.D., N.F., and B.S.M. performed NGS experiments. N.F., B.S.M., I.O., G.D., J.S., S.H.S., Y.W., M.S., and R.S. provided NGS services. M.J.S., and A.O. developed the assembly pipeline and performed genome assembly. K.T., and A.K. performed mining of electronic medical records. A.S.GR., M.M.H., M.J.S., B.C., A.O., F.K., A.GS., M.L., E.M.S., V.S., and H.B. analyzed, interpreted, and/or discussed data. A.S.GR., E.M.S., V.S., and H.B. wrote the manuscript. E.M.S., V.S., and H.B. conceived the study. E.M.S., V.S., and H.B. supervised the study. A.GS., E.M.S., V.S., and H.B. raised financial support. Competing interests: Robert Sebra is VP of Technology Development and a stockholder at Sema4, a Mount Sinai Venture. This work, however, was conducted solely at Icahn School of Medicine at Mount Sinai. Data and materials availability: SARS-CoV-2 genome consensus sequences for all study isolates were deposited in GISAID (accession EPI_ISL_414476, EPI_ISL_415151, and EPI_ISL_421348 to EPI_ISL_421435) and GenBank (accession MT370831 to MT370920). The code used to assemble the consensus genome sequences in this study is available online at https:/github.com/misull/COVID_pipe and (10). This study was reviewed and approved by the Institutional Review Board of the Icahn School of Medicine at Mount Sinai (protocols: HS\#13-00981 and HS\#1601215). This work is licensed under a Creative Commons Attribution 4.0 International (CC BY 4.0) license, which permits unrestricted use, distribution, and reproduction in any medium, provided the original work is properly cited. To view a copy of this license, visit https://creativecommons.org/licenses/by/4.0. This license does not apply to figures/photos/artwork or other content included in the article that is credited to a third party; obtain authorization from the rights holder before using such material.

\section{SUPPLEMENTARY MATERIALS}

science.sciencemag.org/cgi/content/full/science.abc1917/DC1

Materials and Methods

Figs. S1 to S3

Tables S1 and S2

References (11-25)

MDAR Reproducibility Checklist

Data Files S1 and S2

View/request a protocol for this paper from Bio-protocol.

10 April 2020; accepted 27 May 2020

Published online 29 May 2020

10.1126/science.abc1917 


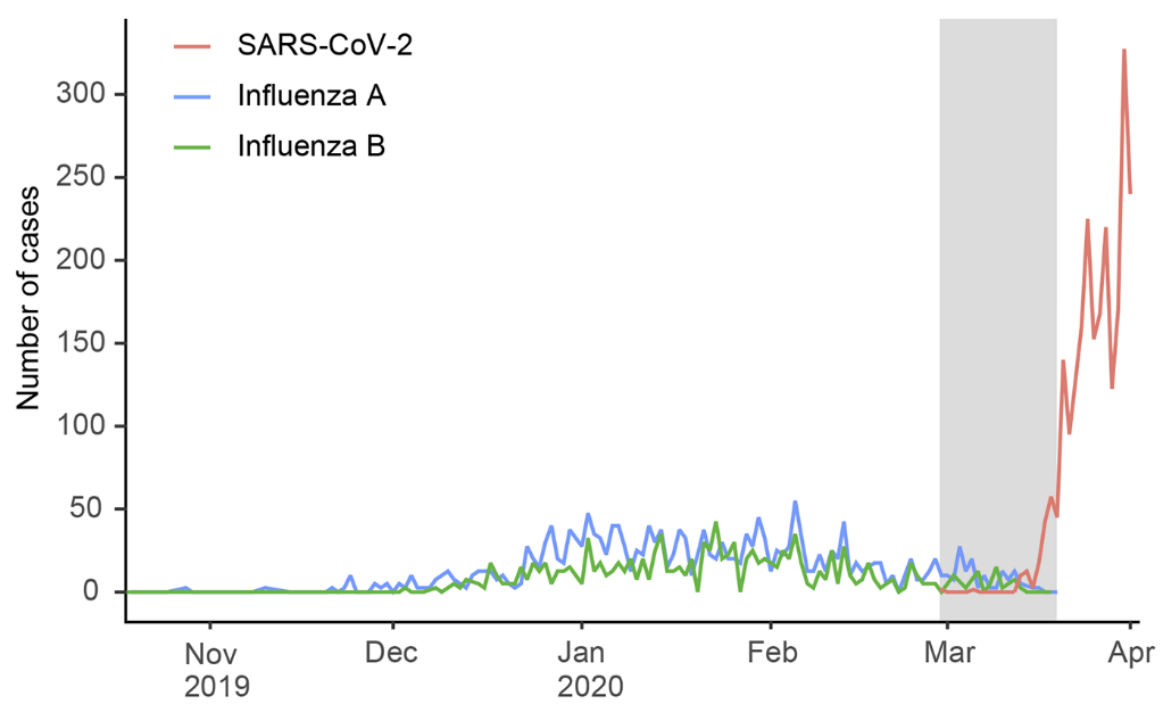

Fig. 1. SARS-CoV-2 confirmed cases. Number of patients with positive molecular tests for SARSCoV-2 up to March 31 2020, compared to the daily number of patients with influenza $A$ and $B$ tests in the 2019-2020 season. The shaded area indicates the period in which the samples sequenced in this study were obtained (February 29th to March 18). The number of positive tests per virus was not normalized for the number of tested samples. 
A

Region (Branches)

- Africa

- Asia

- Europe

- North America

- Oceania

- South America

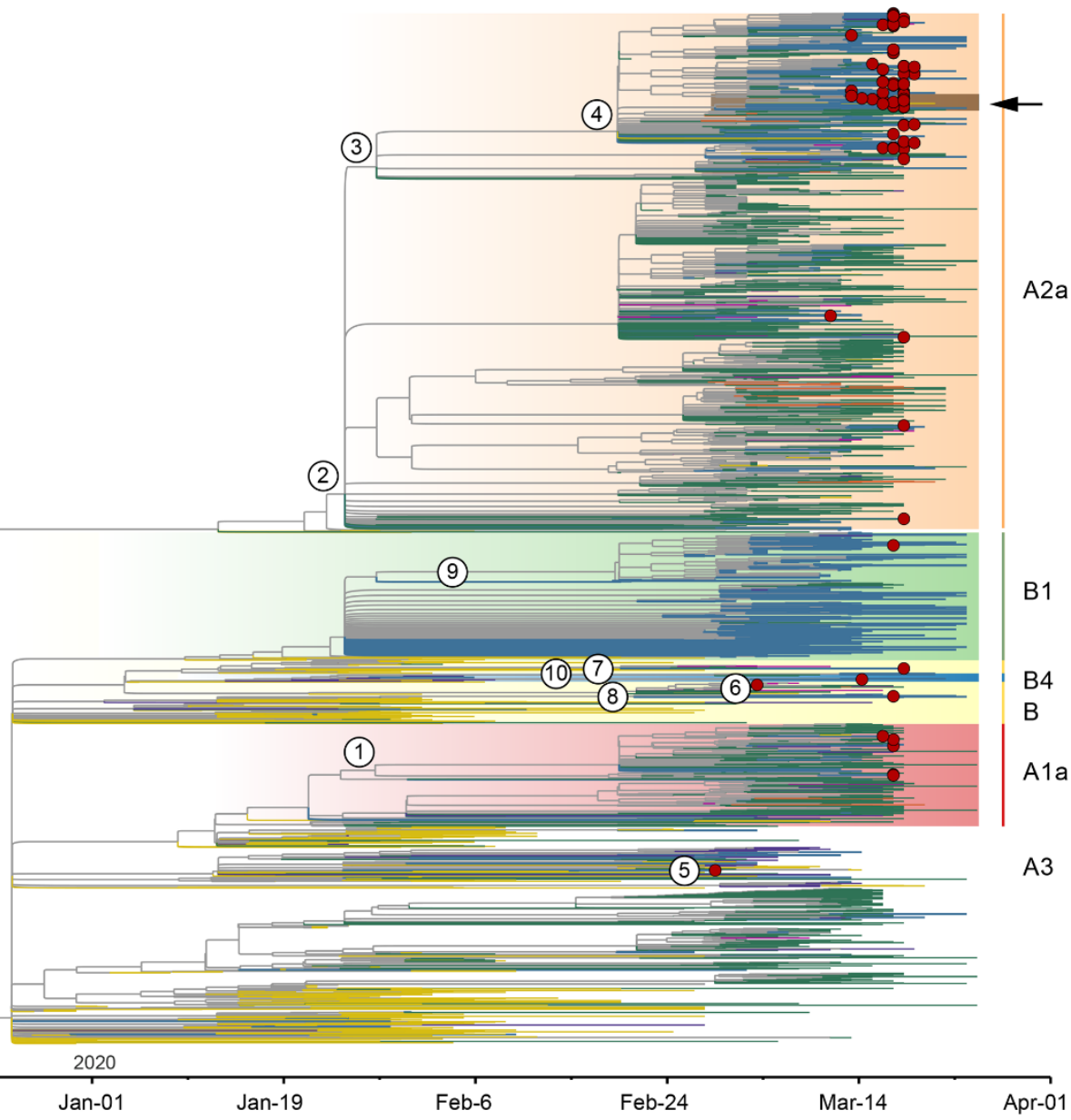

B

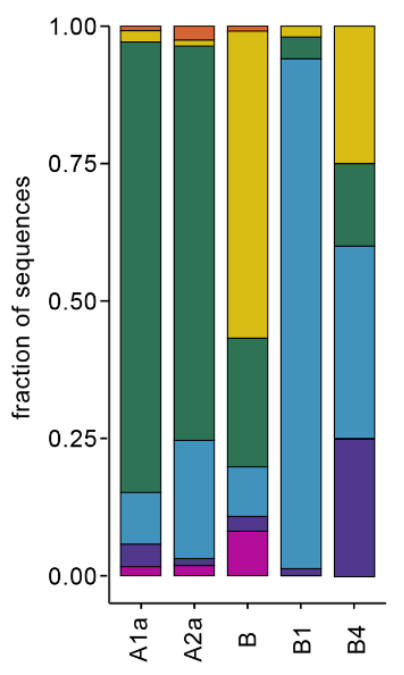

Region

$\square$ Africa $\square$ North America
$\square$ Asia $\square$ Oceania
Europe
South America

C

$$
\begin{aligned}
& \text { Source (Branches) } \\
& \text { - NY (this study) } \\
& \text { - Background }
\end{aligned}
$$

County (tips)
Bronx
Kings
New York
Queens
Westchester

100 ORF1b: A1844V

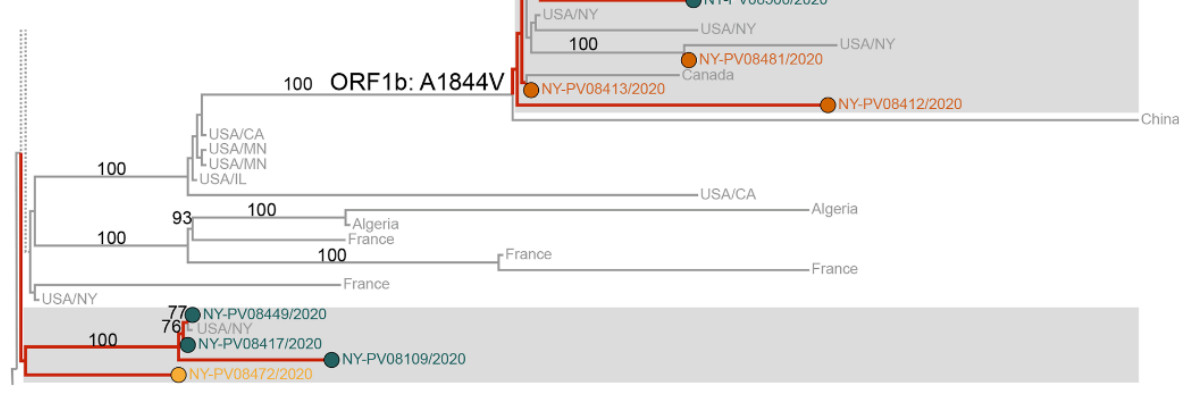


Fig. 2. Phylogenetic relationships of SARS-CoV-2 from NY and other global strains. (A) Maximum Likelihood (ML) phylodynamic inference of 84 SARS-CoV-2 sequences from NY from this study in a global background of 2,363 sequences available in GISAID EpiCoV database as of April 1, 2020. Branches are colored according to the region of origin. Tip circles (red) indicate the position of the $84 \mathrm{NY}$ sequences. Clades that contain NY sequences are highlighted with names shown on the right; the local transmission clusters are indicated by the arrow. The node positions for the transmission events listed in Table 1 are marked by the numbers in white circles. The displayed time tree was inferred under a strict clock model with a fixed substitution rate of $0.8 \times 10-3$. (B) Stacked barplot showing the fraction of sequences per region by clade. (C) Local transmission clusters on the ML tree showing the source of cases by county. Bootstrap support values $\geq 70 \%$ are shown, sibling clusters are collapsed for easier visualization. The mutation identified specific to the community transmission cluster is indicated. The scale bar at the bottom indicates the number of nucleotide substitutions per site. 
A

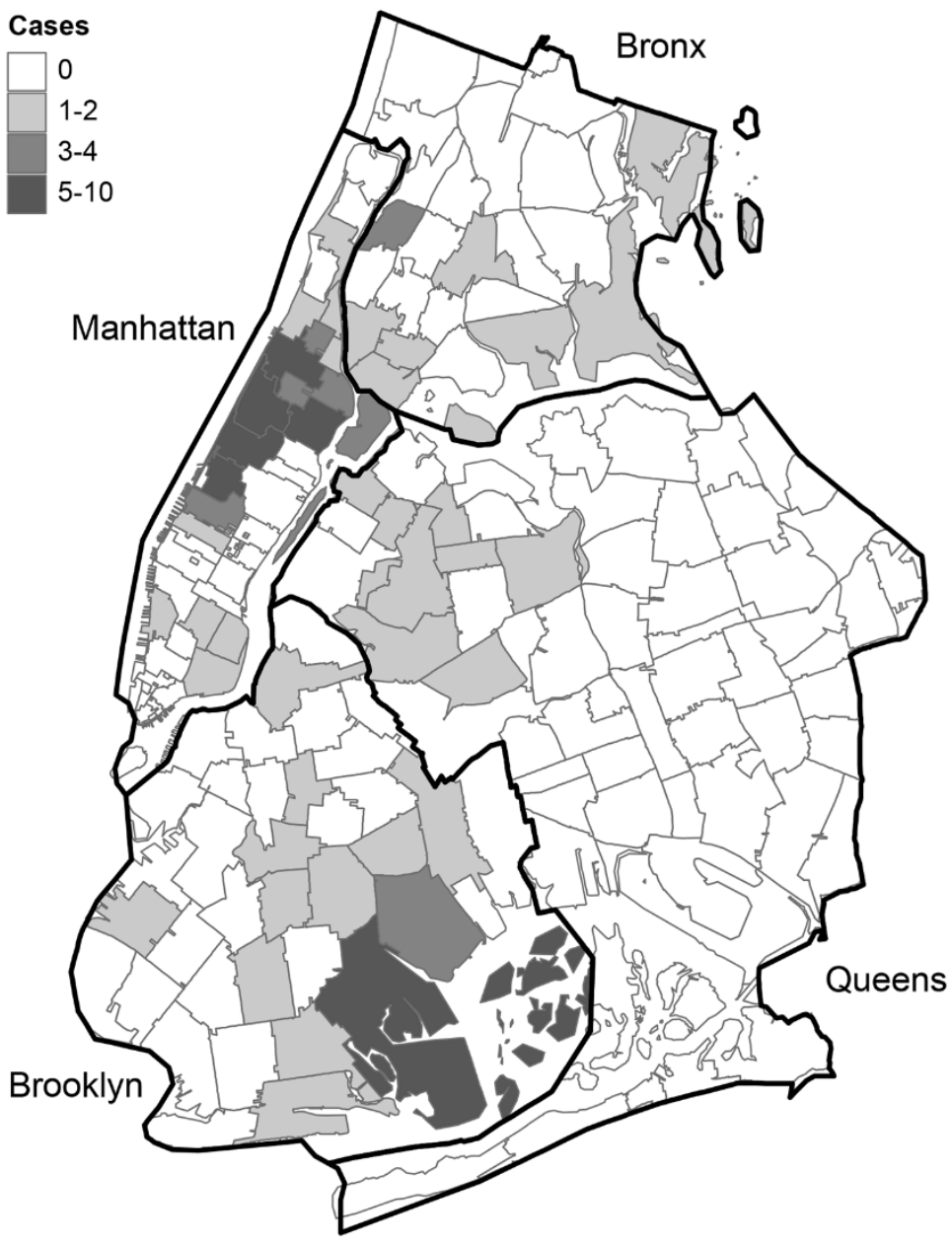

B

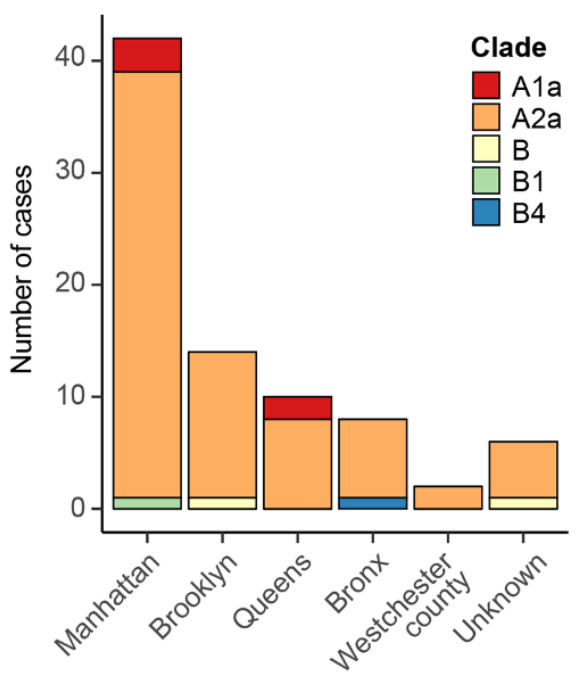

Fig. 3. Distribution of the geographic location of the patients with COVID-19 from which viral isolates were sequenced . (A) Distribution of 74 sequenced cases with available zip code information across NYC boroughs and neighborhoods. Neighborhoods are shaded according to the number of cases that were sampled. (B) Breakdown of sequenced cases according to phylogenetic clades across NYC boroughs and Westchester County. Cases without available zip code information are indicated as 'Unknown'. 
Table 1. Inferred SARS-CoV-2 virus transmission events related to New York City.

\begin{tabular}{|c|c|c|c|c|c|c|c|}
\hline Clade & $\begin{array}{l}\text { First NYC } \\
\text { sequence }\end{array}$ & Mutations & Node & Cases & Event type & Inferred source & tMRCA 95\% HPD* \\
\hline$\overline{\mathrm{A} 1 \mathrm{a}}$ & 4-Mar & $\begin{array}{l}\text { ORF3a:G251V, } \\
\text { C14805T }\end{array}$ & 1 & 5 & Putative introduction & Europe & Jan $25-$ Feb 6 \\
\hline \multirow[t]{3}{*}{$\mathrm{A} 2 \mathrm{a}$} & 11-Mar & $\begin{array}{l}\text { S:D624G, } \\
\text { ORF1b:P314L }\end{array}$ & 2 & 4 & $\begin{array}{l}\text { Untracked transmis- } \\
\text { sion }\end{array}$ & N/A & Jan $5-$ Jan 29 \\
\hline & & $\begin{array}{l}\text { S:D624G, } \\
\text { ORF1b:P314L, } \\
\text { OFR3a:Q57H }\end{array}$ & 3 & 5 & Putative introduction & Europe / North America & Jan $31-$ Feb 11 \\
\hline & & $\begin{array}{l}\text { S:D624G, } \\
\text { ORF1b:P314L, } \\
\text { OFR3a:Q57H, } \\
\text { ORF1a:T265I }\end{array}$ & 4 & 64 & Putative introduction & Europe / North America & Feb $15-$ Feb 19 \\
\hline A3 & 29-Feb & ORF1a:V378I & 5 & 1 & $\begin{array}{l}\text { Travel } \\
\text { exposure }\end{array}$ & Middle East (epi link) & $\begin{array}{l}\text { Feb } 29 \\
\text { (Test date) }\end{array}$ \\
\hline \multirow[t]{3}{*}{ B } & 4-Mar & ORF8:L84S & 6 & 1 & $\begin{array}{l}\text { Travel } \\
\text { exposure }\end{array}$ & Europe (epi link) & $\begin{array}{l}\text { Mar } 4 \\
\text { (Test date) }\end{array}$ \\
\hline & & & 7 & 1 & Putative introduction & Europe / Asia & Feb $2-$ Feb 22 \\
\hline & & & 8 & 1 & Putative introduction & North America / Domestic & Jan $28-$ Feb 24 \\
\hline B1 & 17-Mar & $\begin{array}{l}\text { C18060T, } \\
\text { ORFb:P1427L }\end{array}$ & 9 & 1 & Putative introduction & Domestic & Jan $26-$ Feb 7 \\
\hline B4 & 14-Mar & $\begin{array}{l}\text { N:S202N, } \\
\text { ORF14:V94I }\end{array}$ & 10 & 1 & Putative introduction & Asia / Oceania & Jan $31-$ Feb 25 \\
\hline
\end{tabular}

*Inferred 95\% High Posterior Density (HPD) interval for the mean time of the most recent common ancestor (tMRCA) from the root of the node containing NY-origin strains with $\geq 70 \%$ bootstrap support value in the ML tree and $\geq 0.9$ posterior probability in the Maximum Clade Credibility (MCC) trees inferred for major clades. The 95\% HPD intervals shown correspond to the MCC trees inferred using a strict clock model. Travel exposures and putative introductions are shown for the cases described in this study. 